\title{
Overweight and Obesity Categories of Residents in A Local Urban Area
}

\author{
R.S Tshalibe,N. Ndlovu , T. Usai,Makamure C.,Chinofunga D., B.Mutonhodza \\ and S.T. Kawadza \\ ${ }^{I}$ (Department of Food Science and Nutrition, Midlands State University, P. Bag 9055 Gweru, Zimbabwe)
}

\begin{abstract}
The purpose of this study was to determine the applicability of the WHO categories of overweight and obesity in Gweru residents. Measurements of body mass index (BMI), waist circumference and skin fold were carried out. The manifestation of either hypertension, type 2 diabetes or both in individuals were self reported. Findings revealed that hypertension and Type 2 diabetes were prevailing at significantly higher BMI categories in Gweru residents. The number of individuals who were obese was significantly higher posing them at an increased risk of the consequences sooner or later. The researcher recommends intervention that will increase the population's awareness on how to prevent obesity.
\end{abstract}

Keywords: obesity, BMI, hypertension, type 2 diabetes, nutrition

\section{INTRODUCTION}

According to Kuczmarski and Flegal [1], overweight and obesity are ranges that depict excessive fat accumulation in the body that may impair health. The WHO definition [2] for overweight is a BMI greater than or equal to 25 , while that for obesity is a BMI greater than or equal to 30 . Assessment and classification of these conditions are dependent on specific BMI. BMI is a simple index of weight for height that is used to classify whether a person is underweight, normal, overweight or obese in adults. It provides the most useful measure of body fat composition as BMI is the same for both sexes and for all ages of adults. However, it may not correspond to the same degree of fatness in different individuals [3].

Globally, the prevalence of overweight and obesity has increased since 1980. However, estimates of trends in overweight and obesity prevalence by country are not available [4]. A raised BMI is associated with the prevalence of obesity related disorders such as Type 2 diabetes, hypertension, high cholesterol levels in the blood leading to cardio-vascular diseases (CVDs) mainly stroke, heart disease, hernias, infertility and metabolic syndrome [5]. Raised BMI is also a major risk factor for musculo-skeletal disorders and some cancers which cause poor quality of life and eventually death [6].

As evidence towards the fact that the WHO guidelines may not be applicable to all populations, previous studies have shown that in Asian populations, comorbidities associated with obesity related diseases occur at a much lower BMI than in individuals of either the European or American descent [7]. This was attributed to the high visceral or abdominal obesity in these populations that puts them at a higher risk for obesity related disorders at a lower BMI. This led to WHO reassessing the BMI classification criteria for Asians with the obesity task force lowering the BMI cutoffs to 23 for overweight and 25 for obesity [8]. Researchers have also reported that Filipino Americans have higher rates of metabolic syndrome, insulin resistance and greater intra-abdominal visceral adiposity than blacks and whites [9], hence the need to also have their BMI cut offs lowered.

In contrast, Pacific Islanders appear to be more muscular and have comparably low levels of body fat at a given BMI [4]. Hence higher cutoff points were suggested for this population which are a BMI greater than or equal to 26 for overweight and greater than or equal to 32 for obesity.

The major drawback of BMI as an index for assessing risk of obesity related disorders is that it does not give an indication of body composition based on the amount or distribution of fat in the body [10]. BMI standard was set using data primarily from whites of western origin [11]. The differences in body composition have been noted between these whites and other populations. At an identical BMI, Africans have lower intraabdominal visceral adiposity than their European counterparts. Africans also have higher levels of HDL and a corresponding lower serum triglyceride level as compared to their white counterparts [7].

This study seeks to identify the BMI range at which metabolic diseases begin to show prevalence in a local town community in Zimbabwe. The study further seeks to assess the knowledge of individuals as far as BMI is concerned. It intends to elaborate on the applicability of the WHO definitions of overweight and obesity in Gweru residents.

Previous studies done in some areas in Zimbabwe show that obesity related disorders were occurring at lower BMI categories than those proposed by WHO [12]. The statistics for sudden deaths in Zimbabwe due to 
obesity were at $15.7 \%$ according to the Statesman [13]. There are no reports of trends on the prevalence of obesity in Zimbabwe hence the need to carry out this study.

\subsection{Anthropometric measurements}

\section{METHOD}

Measurements of body mass index, waist circumference and skin fold were carried out on 60 individuals ( $\geq 21$ years) from Gweru urban low, middle and high income groups.

\subsection{Interviews}

Assessment of knowledge on BMI, susceptibility to obesity related disorders and other information sought by the researcher were obtained using interviews for all the 60 participants.

\subsection{Questionnaire}

Qualitative food frequency questionnaires were used to obtain the usual number of times foods on a checklist were consumed over a week for the 60 participants.

\section{RESULTS AND DISCUSSION}

\subsection{Prevalence of hypertension}

Table 1 gives a summary for the prevalence of hypertension in males and females. In males, hypertension begins to prevail in the very obese BMI category and at a waist circumference greater than $102 \mathrm{~cm}$. Prevalence of hypertension in female occurs in the obese range and at a waist circumference greater than $88 \mathrm{~cm}$. There are no males (M) or females (F) experiencing hypertension in the overweight range. According to WHO guidelines, hypertension is expected to begin occurring in overweight individuals. Application of statistical chi square test showed that there is a significant difference in the prevalence of Type 2 hypertension in Gweru residents as compared to the WHO BMI standards.

Table 1: Prevalence of hypertension

\begin{tabular}{|l|c|c|c|c|c|c|}
\hline \multirow{2}{*}{ BMI } & \multicolumn{2}{|c|}{ Number } & \multicolumn{2}{c|}{$\begin{array}{l}\text { Number with } \\
\text { hypertension }\end{array}$} & \multicolumn{2}{c|}{$\begin{array}{l}\text { Waist circumference at which hypertension } \\
\text { prevails (cm) }\end{array}$} \\
\cline { 2 - 7 } & M & F & M & F & M & F \\
\hline $25-29.9$ (overweight) & 7 & 12 & 0 & 0 & 0 & 0 \\
\hline 30 (obese) & 3 & 11 & 0 & 4 & 0 & $>88$ \\
\hline 40 (very obese) & 3 & 0 & 1 & 0 & 102 & 0 \\
\hline
\end{tabular}

\subsection{Prevalence of Type 2 diabetes}

The Table 2 shows the prevalence of type 2 diabetes in males and females. Type 2 diabetes begins to prevail in the very obese males at a waist circumference greater than $102 \mathrm{~cm}$. The prevalence of Type 2 diabetes is shown in 1 female who is in the obese BMI range and has a waist circumference greater than $88 \mathrm{~cm}$. There are no males or females experiencing diabetes in the overweight range. Application of statistical chi square test showed that there is a significant difference in the prevalence of Type 2 diabetes in Gweru residents as compared to the WHO BMI standards.

Obese related disorders started at BMI greater than 29. Similar results have been noted in the studies carried out for Pacific Islanders where obesity related disorders began to show prevalence at a BMI of 32. These results may be attributed to the fact that Africans have lower abdominal fat, a greater bone density and more lean muscle as compared to their European counterparts as highlighted earlier by Wagner and Heyward [13].

Table 2: prevalence of Type 2 diabetes

\begin{tabular}{|c|c|c|c|c|c|c|}
\hline \multirow[t]{2}{*}{ BMI } & \multicolumn{2}{|c|}{ Number } & \multicolumn{2}{|c|}{$\begin{array}{l}\text { Number with } \\
\text { diabetes }\end{array}$} & \multicolumn{2}{|c|}{$\begin{array}{l}\text { Waist circumference at which diabetes } \\
\text { prevails }(\mathrm{cm})\end{array}$} \\
\hline & $\mathrm{M}$ & $\mathrm{F}$ & $\mathrm{M}$ & $\mathrm{F}$ & $\mathrm{M}$ & $\mathrm{F}$ \\
\hline $25-29.9$ (overweight) & 7 & 12 & 0 & 0 & 0 & 0 \\
\hline 30 (obese) & 3 & 11 & 0 & 1 & 0 & $>88$ \\
\hline 40 (very obese) & 3 & 0 & 2 & 0 & 102 & 0 \\
\hline
\end{tabular}

\subsection{Anthropometric measures}

\subsubsection{BMI}

Fig. 1 shows that $47.3 \%$ of individuals who are in the normal BMI range (18.5-24.9) are from the high income status category. $41 \%$ is depicted for the overweight (25-29.9) range from individuals with low income status. $63.6 \%$ of the individuals who were obese also had a low income status, while $66.7 \%$ of the very obese people also had a low income status. 


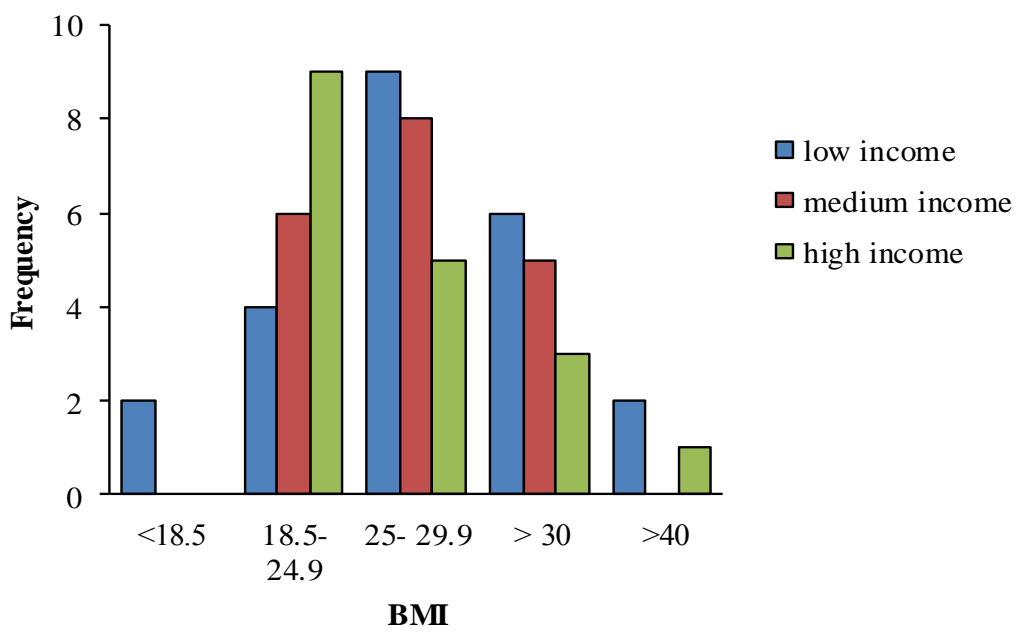

Figure 1: BMI categories of participants in different income levels

\subsubsection{Skin fold measures}

Fig. 2 shows body fat status of the respondents based on skin fold measurements. Most people had ideal body fat percentages denoted by $49.1 \%$, while those who were average were at $42.1 \%$. The obese category presented a $5.26 \%$, while the least category was the lean which had $3.5 \%$.

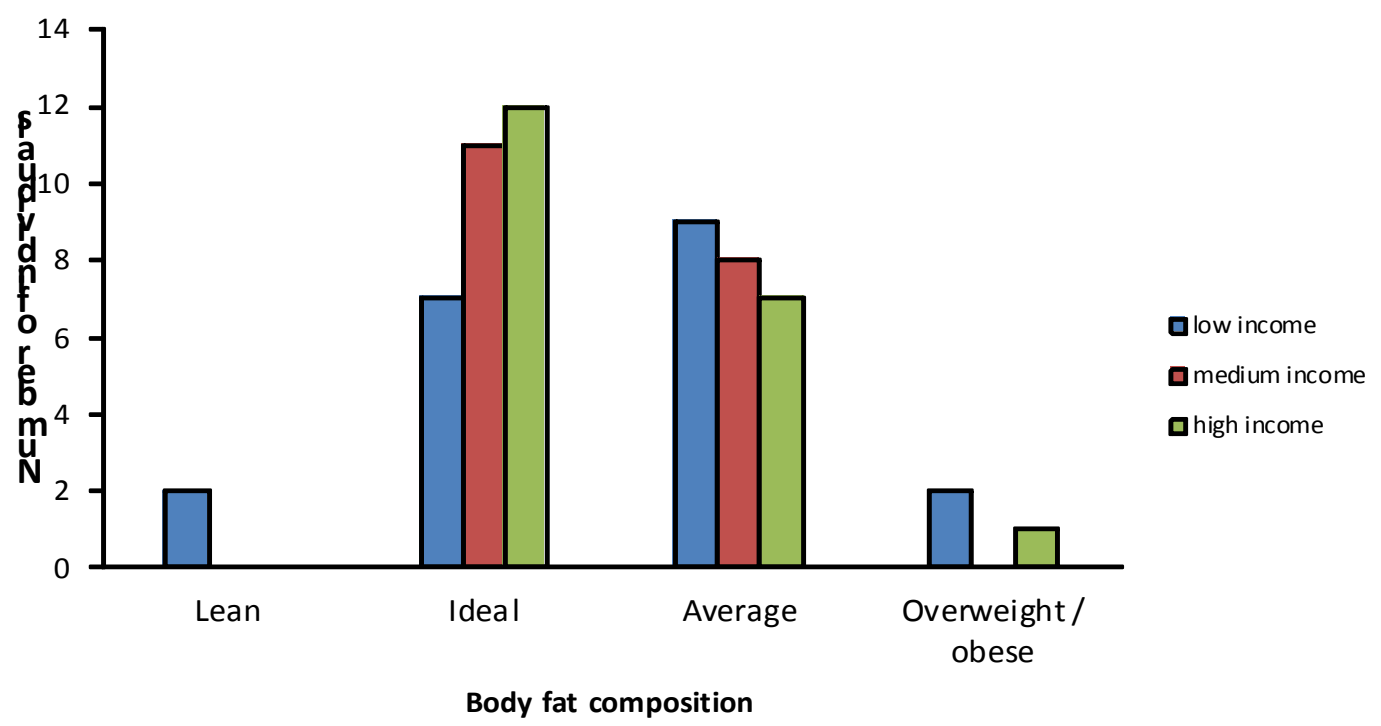

Figure 2: Graph showing body fat status using skin fold measures

\subsubsection{Knowledge on BMI}

The respondents' knowledge on BMI is shown in Fig. 3. Knowledge on BMI was highest in the high income individuals probably attributed to access to the internet, media and higher educational levels. BMI knowledge in low income group was least probably due to limited access to reading materials and media. 


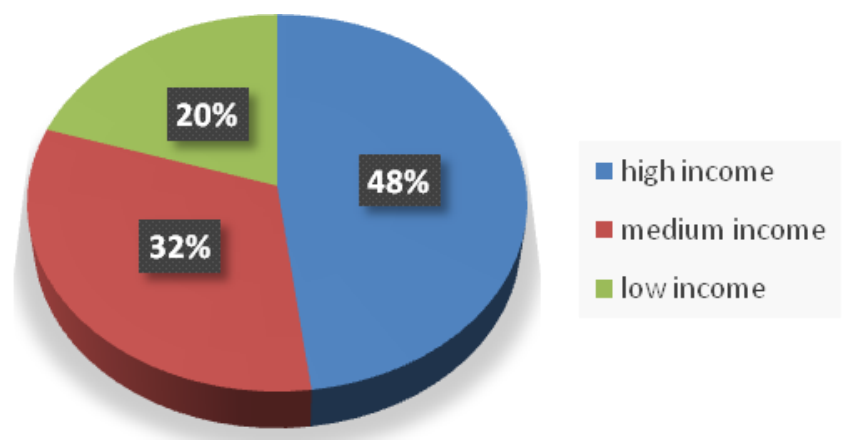

Figure 3: Knowledge on BMI in different income categories

\subsection{Nutrition}

\subsubsection{Dietary assessments}

Fig. 4 shows food frequency distribution for all income earners. Low income group consumed a diet that was high in fat and carbohydrate since they could afford expensive healthy food. Middle income group consumed moderate amounts of fats and carbohydrates. High income group consumed a healthy diet rich in fruits, vegetables and proteins.

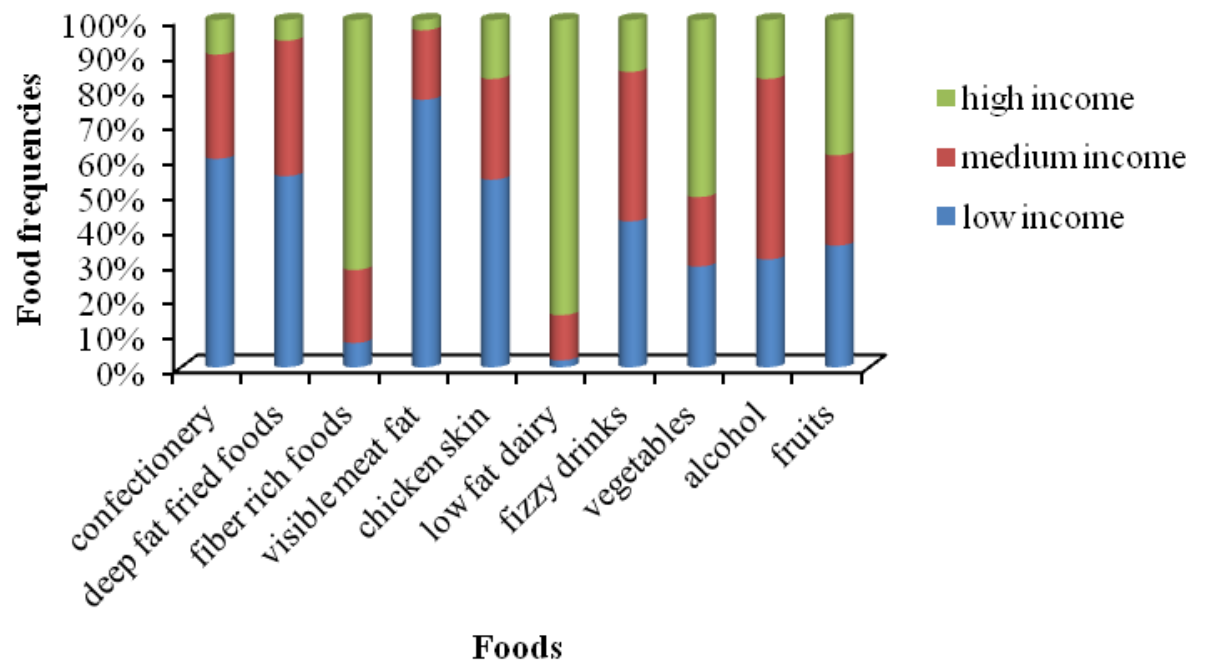

Figure 4: Foods and beverages consumed over a week

\section{CONCLUSION}

Results from the study enabled the researcher to fairly conclude that obesity related disorders were occurring at BMI levels that were significantly higher than the overweight and obese level ranges. This was attributed to the differences in the fat patterning, bone density, bone mineral content and ratio of lean tissue between blacks and whites, having blacks with higher bone densities and lower intra-abdominal obesity which poses a higher risk for hypertension and Type 2 diabetes. These findings do not agree with WHO guidelines: hypertension is expected to begin occurring in overweight individuals. Application of statistical chi square test showed that there is a significant difference in the prevalence of Type 2 hypertension in Gweru residents as compared to the WHO BMI standards. Further research on the investigation at national level would give results which are more conclusive.

\section{Acknowledgements}

The authors would like to acknowledge the cooperation from Gweru residents for providing valuable information that enabled this study to be successful. 


\section{REFERENCES}

[1] R.J. Kuczmarski and M.F. KatherineCriteria for definition of overweight in transition: background recommendations, The American Journal of Clinical Nutrition, 72(5), 2000, 1074- 1081.

[2] B. Balkau, M.A. Charles, T. Drivsholm, K. Borch-Johnsen and N. Wareham, Frequency of the WHO metabolic syndrome in European cohorts, and an alternative definition of an insulin resistance syndrome, Diabetes \&Metabolism, 28(5), 2002, 364-376.

[3] J.C Seidell , A.Oosterlee,P. Deurenberg , J.G.Hautvast , J.H.Ruijs , Abdominal fat depots measured with computed tomography: effects of degree of obesity, sex, and age, European Journal of Clinical Nutrition, 42(9), 1988, 805-815.

[4] S.V.Hubbard, Defining overweight and obesity: what are the issues? American Journal of Clinical Nutrition, 72(5), 2000, 10671068 .

[5] S.C. Savva, M. Tornaritis, M.E. Savva, Y. Kourides, A. Panagi1, N. Silikiotou, C. Georgiou and A. Kafatos, Waist circumference and waist-to-height ratio are better predictors of cardiovascular disease risk factors in children than body mass index, International Journal of Obesity, 24, 2000 1453-1458.

[6] J.C. Stevenson, A woman's journey through the reproductive, transitional and postmenopausal periods of life: impact on cardiovascular and musculo-skeletal risk and the role of estrogen replacement, Maturitas, 70(2), 2011, 197-205.

[7] H.A. Tyroler, C.G. Hames, I. Krishan and S. Heyden, Black-white differences in serum lipids and lipoproteins in Evans County, Preventive Medicine, 4(4), 1975, 541-549.

[8] M. Kanazawa, N. Yoshiike, T. Osaka, Y. Numba, P.Zimmet and-S.Inoue, Criteria and classification of obesity in Japan and AsiaOceania, Criteria and classification of obesity in Japan and Asia-Oceania, 94, 2005, 1-12.

[9] S.C. Smith, L.T. Clark, R.S. Cooper, and S.R. Daniels, Discovering the Full Spectrum of Cardiovascular Disease Minority Health Summit 2003: Report of the Obesity, Metabolic Syndrome, and Hypertension Writing Group, Circulation, American Heart Association, 111, 2005, e134-e139.

[10] F. Bacha, R. Saad, N. Gungor, J. Janosky Obesity, regional fat distribution, and syndrome X in obese black versus white adolescents: race differential in diabetogenic and atherogenic risk factors, Journal of Clinical Endrocrinology and Metabolism, $88(6), 2003,2534-2540)$

[11] E.M Evans, D.A Rowe, S.B Racette, K.M Ross, E. McAuley, Is the current BMI obesity classification appropriate for black and white postmenopausal women?, International journal of Obesity, 30, 2006, 837-843.

[12] W. Siffert, P. Forster, K.H. Jöckel, D.A. Mvere, Worldwide ethnic distribution of the G protein $\beta 3$ subunit $825 \mathrm{~T}$ allele and its association with obesity in Caucasian, Chinese, and Black African individuals, Journal of the American Society Nephrology, 10(9), 1999, 1921-1930.

[13] Statesman Year Book, (McMillan, New York, 2007).

[14] P.R. Wagner and V.H. Heyward, Measures of body composition in blacks and whites: a comparative review, American Journal of clinical Nutrition, 98(3), 2000 1938-3207. 